\title{
$\lambda$ stenting: a novel technique for posterior communicating artery aneurysms with fetal-type posterior communicating artery originating from the aneurysm dome
}

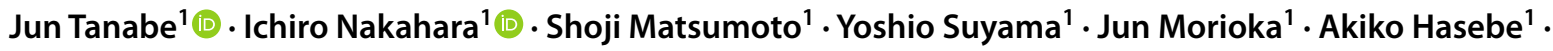 \\ Sadayoshi Watanabe ${ }^{1} \cdot$ Kenichiro Suyama $^{1} \cdot$ Kiyonori Kuwahara $^{1} \cdot$ Keiko Irie $^{2}$
}

Received: 16 May 2021 / Accepted: 19 July 2021 / Published online: 5 August 2021

(C) The Author(s) 2021

\begin{abstract}
Purpose Endovascular treatment of posterior communicating artery aneurysms with fetal-type posterior communicating artery originating from the aneurysm dome is often challenging because, with conventional techniques, dense packing of aneurysms for posterior communicating artery preservation is difficult; moreover, flow-diversion devices are reportedly less effective. Herein, we describe a novel method called the $\lambda$ stenting technique that involves deploying stents into the internal carotid artery and posterior communicating artery.

Methods Between January 2018 and September 2020, the $\lambda$ stenting technique was performed to treat eight consecutive cases of aneurysms. All target aneurysms had a wide neck (dome/neck ratio $<2$ ), a fetal-type posterior communicating artery with hypoplastic P1, and a posterior communicating artery originating from the aneurysm dome. The origin of the posterior communicating artery from the aneurysm, relative to the internal carotid artery, was steep $\left(<90^{\circ}: \mathrm{V}\right.$ shape).

Results The maximum aneurysm size was $8.0 \pm 1.9 \mathrm{~mm}(6-12 \mathrm{~mm})$. The average packing density (excluding one regrowth case) was $32.7 \pm 4.2 \%(26.8-39.1 \%)$. Initial occlusion was complete occlusion in $6(75.0 \%)$ patients and neck remnants in 2 (25.0\%) patients. Follow-up angiography was performed at $18.4 \pm 11.6$ months (3-38 months). There were no perioperative complications or reinterventions required during the study period.

Conclusion The $\lambda$ stenting technique enabled dense coil packing and preservation of the posterior communicating artery. This technique enabled safe and stable coil embolization. Thus, it could become an alternative treatment option for this sub-type of intracranial aneurysms.
\end{abstract}

Keywords Aneurysm $\cdot$ Posterior communicating artery $\cdot$ Endovascular $\cdot$ Coiling $\cdot$ Stent

\section{Introduction}

Posterior communicating artery (PCOM) aneurysms constitute approximately $30 \%$ of all aneurysms [1]. Endovascular therapy remains the first-line treatment, especially for ruptured or posterior circulation aneurysms [2, 3]. Moreover, many PCOM aneurysms are treated safely and effectively by

Jun Tanabe

j.tanabe@fujita-hu.ac.jp

1 Department of Comprehensive Strokology, Fujita Health University School of Medicine, 1-98 Dengakugakubo, Kutsukake-cho, Toyoake, Aichi, Japan

2 Department of Neurosurgery, Nagoya Kyoritsu Hospital, 1-172 Hokke, Nakagawa-ku, Nagoya, Aichi, Japan endovascular coiling. Although a fetal-type PCOM itself is not a risk factor, PCOM aneurysms with a fetal-type PCOM "originating from the aneurysm dome" are a risk factor for initial incomplete embolization and long-term instability $[4,5]$. Dense packing of the aneurysm and preservation of the PCOM is difficult using a conventional stent and/ or balloon-assisted adjunctive techniques. Furthermore, flow diversion devices have been reported to be less effective for the treatment of fetal-type PCOM aneurysms [6, 7]. However, drastic advancements in endovascular therapy have enabled stent deployment in the PCOM. Herein, we describe a novel method called "the $\lambda$ stenting technique" that involves deploying stents to the internal carotid artery (ICA) and the PCOM, thereby creating a lambdoid-shaped stent to achieve a relatively dense packing of aneurysms and successful preservation of the PCOM. 


\section{Methods}

\section{Clinical application}

The present study is a retrospective review of medical charts and radiographic data. The study protocol was conducted in accordance with the principles of the Declaration of Helsinki and approved by the institutional ethics committee. The need for written informed consent for participation was waived in accordance with the national legislation and institutional requirements. On our institute's website, all participants were provided with the opportunity to opt out of this study. Decisions for radical treatment modalities were made jointly by neurological and endovascular surgeons. Between January 2018 and September 2020, the $\lambda$ stenting technique was performed to treat eight consecutive cases of aneurysms. The criteria to target aneurysm in the present study were as follows: all target aneurysms had wide neck (dome/neck ratio $<2$ ), a fetal-type PCOM with hypoplastic P1, a PCOM caliber > the P1 segment of the posterior cerebral artery, and a PCOM originating from the aneurysm dome. The PCOM origin from the aneurysm, relative to the ICA, was steep $\left(<90^{\circ}\right.$ : V shape). The aneurysm volume was calculated using a 3D-rotational angiography workstation (Canon Medical Systems, Japan; Philips Medical Systems, the Netherlands). The coil volume was calculated using the following equation:

Coil volume $=\pi \times(\text { diameter of coil } / 2)^{2} \times$ length of coil
The packing density was calculated as the ratio of the coil volume to the aneurysm volume.

\section{Technical details}

All procedures were performed under general anesthesia. Perioperative dual antiplatelet management (aspirin, 100 $\mathrm{mg}$ /day; clopidogrel, $75 \mathrm{mg} /$ day) was administered at least 14 days before the procedure. The period of postoperative antiplatelet therapy depended on the follow-up angiographic result and the discretion of the attending physician. In general, the dual antiplatelet therapy was maintained for 6 months after the procedure; a single agent was prescribed indefinitely thereafter. Systemic heparinization was maintained with an activated clotting time of at least $250 \mathrm{~s}$ during the procedure.

Technical details of the $\lambda$ stenting technique are presented in Fig. 1. A large-bore guiding catheter (an 8-Fr guiding catheter or a 7-Fr guiding sheath), which accepts three microcatheter systems, was placed at the cervical internal carotid artery. The tip of a pre-shaped microcatheter, such as the Excelsior SL10 J in most cases or the C preshaped microcatheter (Stryker, Kalamazoo, MN, USA), or a manually shaped microcatheter, such as a micro pigtail, was directed toward the orifice of the PCOM antegradely. A steerable but flexible micro guide wire with a soft tip, such as Traxcess 14 (MicroVention-Terumo, Tustin, CA, USA), GT wire (MicroVention-Terumo), or Tenrou 1014 (Kaneka Medix, Kanagawa, Japan), was advanced up to the P3 or to a more distal position in the posterior cerebral artery.
Fig. 1 Schematic image of the $\lambda$ stenting technique used for fetal-type PCOM aneurysms (A). A low-profile stent is deployed in the PCOM after insertion of as many coils as possible using the balloon assisted technique (B). A braided stent is deployed in the ICA, covering the neck of the aneurysm $(\mathbf{C})$. In-stent percutaneous transluminal angioplasty is performed to acquire complete stent apposition; the PCOM stent is crushed in the aneurysm neck between the coil mesh and the ICA stent (D). The ICA and PCOM stents form a complex stent in the lambda configuration, namely 'the $\lambda$ stent' (E). PCOM, posterior communicating artery; ICA, internal carotid artery
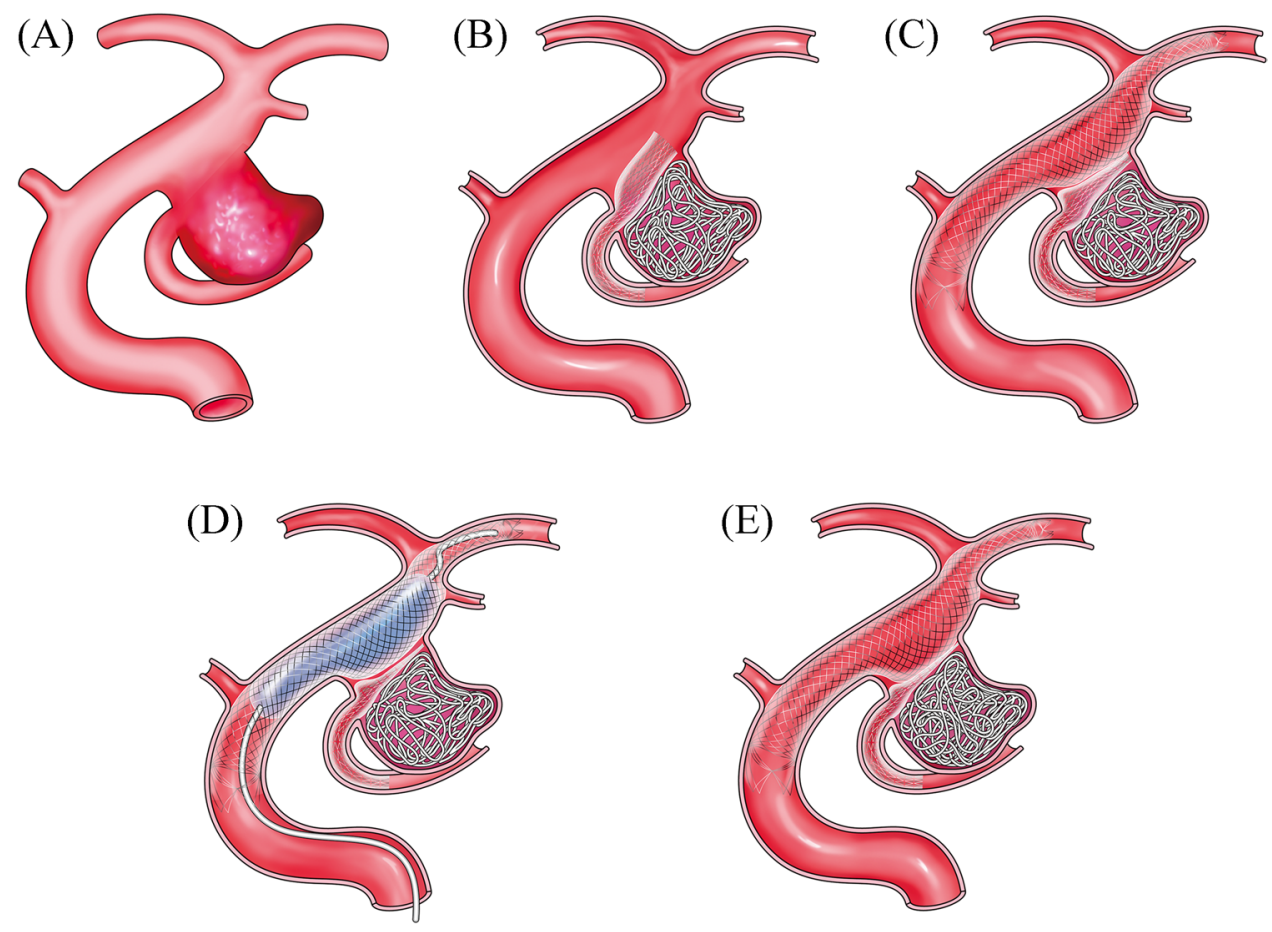
Thereafter, the microcatheter followed the micro guide wire deeply into the PCOM. Before stent deployment, coils were previously inserted through another microcatheter with balloon assistance (mostly Scepter C; $4.0 \mathrm{~mm}$ in diameter, 15 $\mathrm{mm}$ in length; Microvention-Terumo) and catheter assistance of PCOM, while avoiding the protrusion of coils over the ICA and PCOM, respectively. The following stenting procedures are essential to this novel technique. A low-profile short-length open-cell stent (Neuroform Atlas; $3.0 \mathrm{~mm}$ in diameter, $15 \mathrm{~mm}$ in length; Stryker) was deployed from the PCOM to a point slightly distal to the aneurysmal neck, to open the distal flair forward distal neck (Fig. 1B). Subsequently, a braided stent, such as the LVIS (MicroVentionTerumo), was deployed through Headway 21 (exchanged from the balloon catheter or the PCOM microcatheter) in the ICA covering the aneurysm neck using the bulging technique (Fig. 1C). In-stent percutaneous transluminal angioplasty was performed, where necessary, using Scepter or another compliant balloon for complete stent apposition (Fig. 1D). Consequently, the ICA stent crushed a part of the PCOM stent that was placed at the aneurysm neck between the ICA stent and coil mass, creating a complex stent shaped in a lambda configuration, termed " $\lambda$ stenting" (Fig. 1E). Thus, the neck was covered with one layer of the ICA stent and two layers of the PCOM stent, leading to high metal coverage almost equal to the flow diverter at the aneurysm neck. Finally, coil embolization was advanced for a high packing density of the aneurysm, where possible.

\section{Clinical and radiological assessments}

Digital subtraction angiography (DSA) or magnetic resonance angiography (MRA) was recommended at 3,6, and 12 months after coil embolization. Angiographic results were evaluated with the modified Raymond-Roy occlusion classification on DSA or MRA [8]. As the neck of the target aneurysm in the present study was obscure, we considered the neck of these aneurysms as the border between the PCOM stent and the aneurysm wall. Therefore, aneurysms with no filling over PCOM stenting were defined as a modified Raymond-Roy occlusion classification of 1. Perioperative neurological outcomes were evaluated by reviewing the hospital records. Perioperative complications were defined as a deterioration of one point or more in the preoperative modified Rankin scale due to endovascular therapy.

\section{Results}

Eight patients underwent $\lambda$ stenting (Table 1): three men and five women, with a mean age of $63.6 \pm 13.6$ years (45-85 years). Among them, six patients presented with pure unruptured aneurysms, and two patients had regrowth

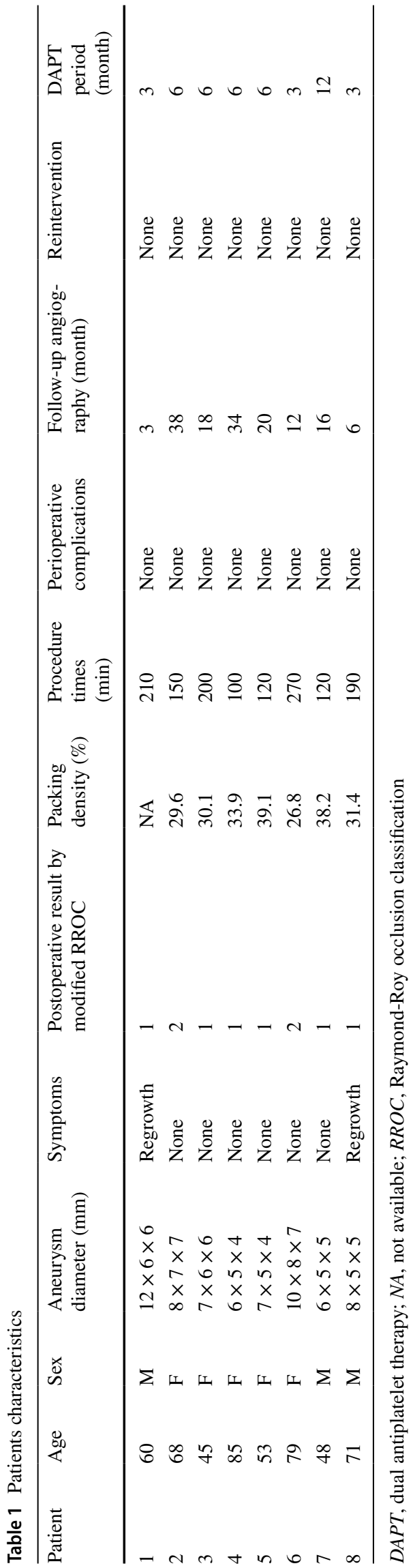


of their aneurysm after previous coiling and clipping, following aneurysm rupture. The maximum aneurysm size was $8.0 \pm 1.9 \mathrm{~mm}(6-12 \mathrm{~mm})$. Successful initial occlusion was achieved in all cases: $6(75.0 \%)$ patients had complete occlusion with a modified Raymond-Roy occlusion classification of 1 and $2(25.0 \%)$ patients had neck remnants with a modified Raymond-Roy occlusion classification of 2 . The average packing density (excluding one case of aneurysm regrowth) was $32.7 \pm 4.2 \%$ (26.8-39.1\%). Furthermore, the average procedure times were $170 \pm 50 \mathrm{~min}$ (100-270 min). Follow-up angiography was performed at $18.4 \pm 11.6$ months (3-38 months). Follow-up angiographic results remained unchanged in all patients. None of the patients experienced perioperative complications or required reintervention during the study period. Dual antiplatelet therapy was maintained at $5.6 \pm 2.8$ months (3-12 months). Thereafter, a single agent was maintained in all patients except 1 case: patient 2 for whom the administration of a single agent was discontinued 3 years after the procedure.

Table 2 shows the technical details of $\lambda$ stenting in each case. In all cases, the transfemoral approach was used. Balloon assistance in the ICA distal to the neck was employed in $3(37.5 \%)$ cases to navigate the microcatheter for the PCOM. A pre-shaped microcatheter (PCOM catheter) was used for PCOM cannulation in $7(87.5 \%)$ cases. In one case (patient 3), a manually shaped microcatheter into a micro pigtail was used. The Neuroform Atlas was utilized for the PCOM stent in all cases. LVIS was utilized for the ICA stent in all cases except one. The in-stent percutaneous transluminal angioplasty was performed using Scepter or SHOURYU (Kaneka Medix, Osaka, Japan) in 6 (75.0\%) cases.

\section{Representative cases}

\section{Case 1}

A 45-year-old woman was diagnosed with an unruptured, large, right PCOM aneurysm (patient 3; Tables 1 and 2; Fig. 2A). An 8-Fr guiding catheter (Roadmaster; Goodman, Aichi, Japan) was navigated to the cervical portion of the right ICA via the right femoral artery. First, a balloon catheter (Scepter XC; $4 \mathrm{~mm} \times 11 \mathrm{~mm}$ ) was positioned in the ICA to cover the aneurysm neck. In addition, a microcatheter for PCOM stenting (Headway 17; MicroVentionTerumo; manually shaped into a micro pigtail) loaded with a micro guide wire (Traxcess 14) was placed in the PCOM without any balloon assistance. Next, a microcatheter to deploy coils was positioned at the aneurysm center. As many coils as possible were inserted using the balloonassisted technique and catheter assistance of PCOM to avoid the protrusion of coils into the ICA and PCOM (Fig. 2B). The balloon catheter was then removed, and a microcatheter for ICA stenting (Headway 21, MicroVention-Terumo) was navigated into the ICA. The Neuroform Atlas $3 \mathrm{~mm} \times 15 \mathrm{~mm}$ was deployed from the PCOM to the distal part of the neck of the aneurysm, and LVIS $4 \mathrm{~mm}$ $\times 22 \mathrm{~mm}$ was deployed to cover the aneurysm neck and crushed the PCOM stent between the ICA stent and the coil mass (Fig. 2C). Headway 21 was exchanged with the $7 \mathrm{~mm} \times 7 \mathrm{~mm}$ SHOURYU for in-stent percutaneous transluminal angioplasty with an exchange wire to deploy the ICA stent. Subsequently, in-stent percutaneous transluminal angioplasty was performed for complete stent apposition. A packing density of $30.1 \%$ and complete obliteration of the aneurysm were achieved without any symptomatic

Table 2 Technical details of $\lambda$ stenting

\begin{tabular}{|c|c|c|c|c|c|c|c|}
\hline Patient & Guiding catheter & $\begin{array}{l}\text { Assist for PCOM } \\
\text { cannulation }\end{array}$ & Balloon catheter & PCOM catheter & PCOM stent & ICA stent & In stent PTA \\
\hline 1 & 8-Fr Roadmaster & None & Scepter C $4 \times 15$ & SL10 J pre-shaped & NF $4.5 \times 15$ & LVIS $4.5 \times 18$ & Scepter C $4 \times 15$ \\
\hline 2 & 7-Fr Shuttle sheath & $\begin{array}{l}\text { Half deployment } \\
\text { of ICA stent and } \\
\text { coils of aneurysm }\end{array}$ & Scepter C $4 \times 15$ & SL10 J pre-shaped & $\mathrm{NF} 3 \times 15$ & LVIS $4.5 \times 23$ & Scepter C $4 \times 15$ \\
\hline 3 & 8-Fr Roadmaster & None & Scepter XC $4 \times 11$ & $\begin{array}{l}\text { Headway } 17 \text { micro } \\
\text { pigtail manual } \\
\text { shaped }\end{array}$ & $\mathrm{NF} 3 \times 15$ & LVIS $4 \times 22$ & $\begin{array}{l}\text { SHOURYU HR } \\
7 \times 7\end{array}$ \\
\hline 4 & 8-Fr Roadmaster & Balloon assist & Scepter C $4 \times 15$ & SL10 C pre-shaped & $\mathrm{NF} 3 \times 15$ & NF $4.5 \times 21$ & none \\
\hline 5 & 8-Fr Roadmaster & None & Scepter C $4 \times 15$ & SL10 J pre-shaped & $\mathrm{NF} 3 \times 15$ & LVIS $4.5 \times 23$ & Scepter C $4 \times 15$ \\
\hline 6 & 8-Fr Roadmaster & None & Scepter C $4 \times 15$ & SL10 J pre-shaped & NF $3 \times 15$ & LVIS $4 \times 22$ & none \\
\hline 7 & 8-Fr Roadmaster & Balloon assist & Scepter C $4 \times 15$ & SL10 J pre-shaped & $\mathrm{NF} 3 \times 15$ & LVIS $4 \times 17$ & $\begin{array}{l}\text { SHOURYU HR } \\
7 \times 7\end{array}$ \\
\hline 8 & 8-Fr Roadmaster & Balloon assist & Scepter XC $4 \times 11$ & SL10 J pre-shaped & $\mathrm{NF} 3 \times 15$ & LVIS $4.5 \times 23$ & Scepter XC $4 \times 11$ \\
\hline
\end{tabular}

PCOM, posterior communicating artery; ICA, internal carotid artery; NF, Neuroform Atlas, SL 10 (Stryker); PTA, percutaneous transluminal angioplasty; Headway 17, Scepter C and XC, LVIS (Terumo); Roadmaster (Goodman); Shuttle sheath (Cook Medical); SHOURYU HR (Kaneka Medix) 
Fig. 2 A representative case. A 3D cerebral angiography image demonstrating a right PCOM aneurysm incorporating the orifice of the PCOM (A). Using the balloon-assisted technique, coil embolization is performed in the dome of the aneurysm after navigating a microcatheter for stent deployment in the PCOM, preserving the ICA and PCOM (B). The ICA and PCOM stents are deployed (C). Postoperative angiography shows complete obliteration of the aneurysm, with PCOM preservation (D). PCOM, posterior communicating artery; ICA, internal carotid artery
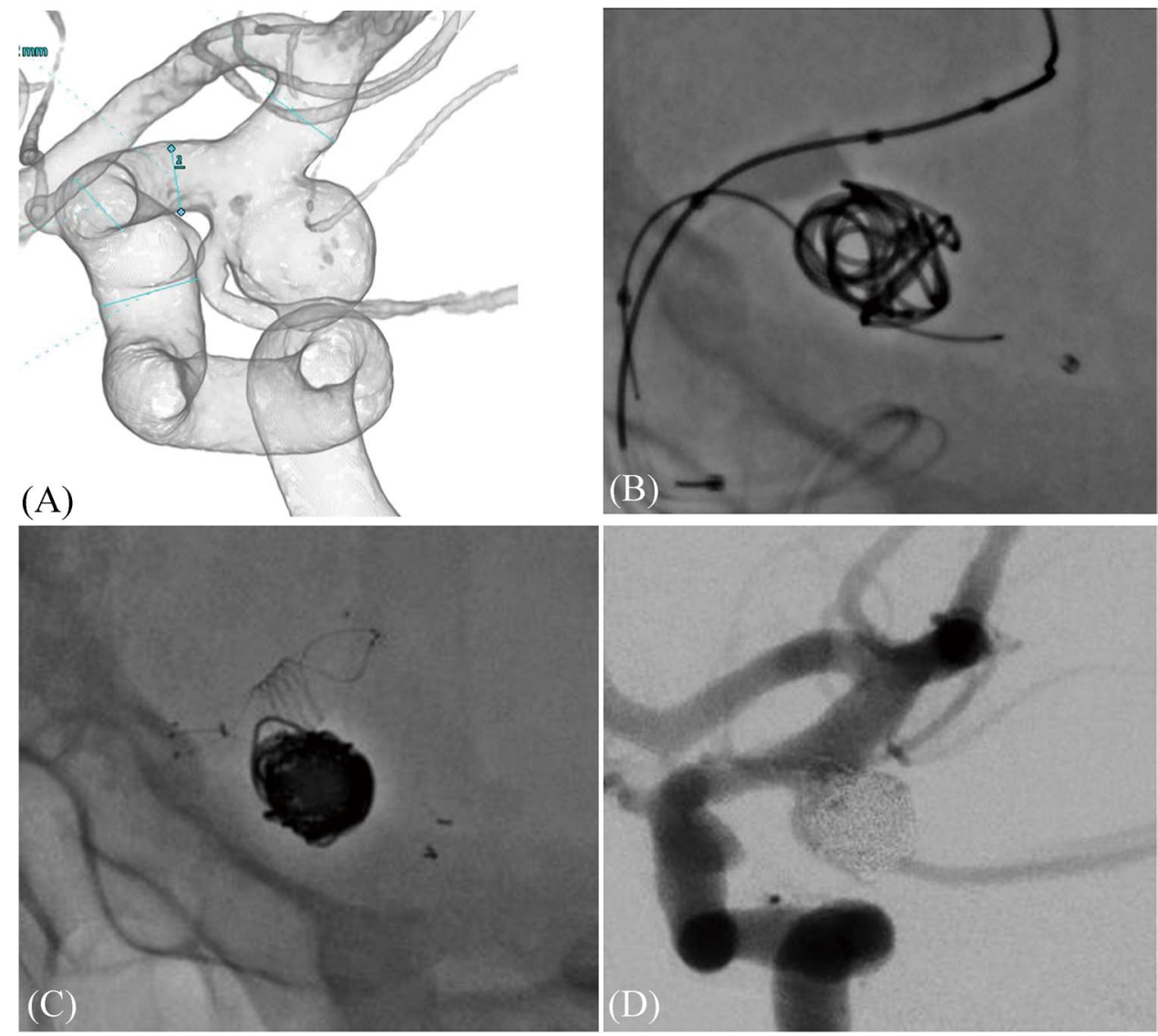

complications (Fig. 2D). Angiographic findings revealed no recurrence 18 months after the procedure.

\section{Case 2}

A 48-year-old man was diagnosed with an unruptured right PCOM aneurysm (patient 7; Tables 1 and 2; Fig. 3A). An 8 -Fr guiding catheter (Roadmaster) was navigated to the cervical portion. First, a balloon catheter (Scepter C; $4 \mathrm{~mm}$ $\times 15 \mathrm{~mm}$ ) was positioned in the ICA to cover the aneurysm neck. In addition, a microcatheter for PCOM stenting (SL10 pre-shaped J), loaded with a micro guide wire (Tenrou 1014), was placed in the PCOM with balloon assistance. Next, balloon-assisted coiling of the aneurysm was performed (Fig. 3B). The balloon catheter was removed, and a microcatheter for ICA stenting (Headway 21) was navigated into the ICA. In advance, ICA stent as LVIS 4 $\times 17 \mathrm{~mm}$ was partially deployed at the ICA distal to the aneurysm to fix the distal end of the ICA stent. Thereafter, the Neuroform Atlas $3 \times 15 \mathrm{~mm}$ was deployed from the PCOM to the distal part of the neck of the aneurysm, and the LVIS was fully deployed to cover the aneurysm neck and crushed the PCOM stent between the ICA stent and the coil mass. In-stent percutaneous transluminal angioplasty was performed for complete stent apposition with SHOURYU
$7 \times 7 \mathrm{~mm}$ (Fig. 3C). A packing density of $38.2 \%$ and complete obliteration of the aneurysm were achieved without any symptomatic complications (Fig. 3D). Angiographic findings revealed no recurrence after 16 months. The operative technique performed for the illustrative case of patient 7 is presented in Supplemental Video.

\section{Discussion}

\section{Principal of $\lambda$ stenting}

The present study describes the safety and efficacy of a newly developed stenting method called the " $\lambda$ stenting technique" for treating PCOM aneurysms with fetal-type PCOM originating from the aneurysm dome. The $\lambda$ stenting technique is characterized by crushing the PCOM stent between the ICA stent and a coil mesh. In the field of coronary intervention, the crush technique has been widely used for coverage of the bifurcation, including the side branch ostium, to prevent restenosis at the ostium of the side branch in the gap between the main branch and side branch stents [9]. Similarly, the $\lambda$ stenting technique is proposed herein for secure coverage of the ostium of the PCOM without a gap between the ICA and PCOM, as the gap often becomes 
Fig. 3 A representative case. A 3D cerebral angiography image demonstrating a right $\mathrm{PCOM}$ aneurysm incorporating the orifice of the PCOM (A). Using the balloon-assisted technique, coil embolization is performed in the dome of aneurysm after navigating a microcatheter for stent deployment in the PCOM, preserving the ICA and PCOM (B). In-stent percutaneous transluminal angioplasty is performed for complete stent apposition after the ICA and PCOM stents are deployed (C). Postoperative angiography shows complete obliteration of the aneurysm, with PCOM preservation (D). PCOM, posterior communicating artery; ICA, internal carotid artery
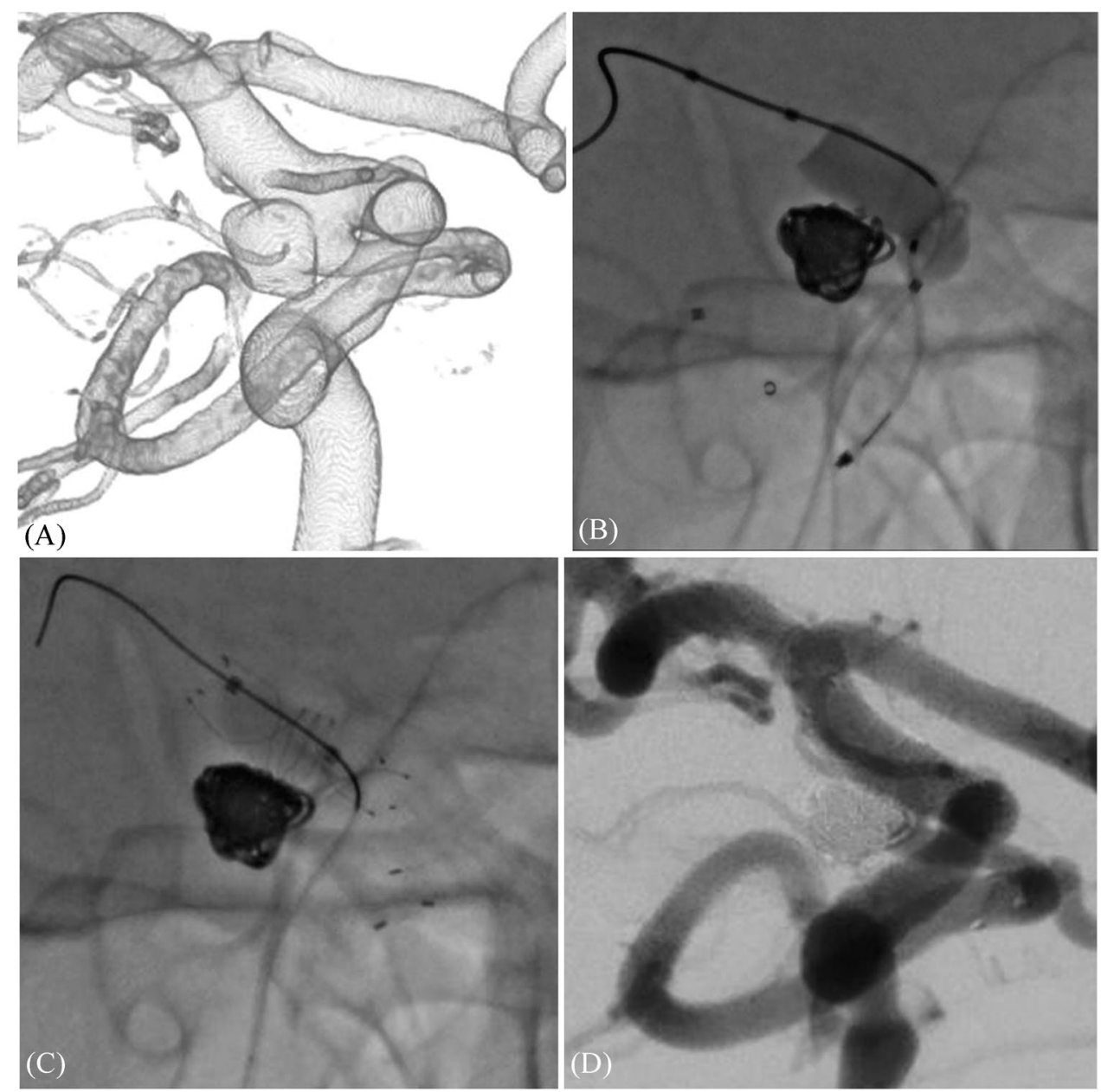

problematic for the below-stated $\mathrm{T}$ configuration stenting technique. Therefore, the $\lambda$ stenting technique can induce high packing density for fetal-type PCOM aneurysms, even for those that have the ostium of the PCOM at their dome. In addition, the $\lambda$ stenting technique, which is a multilayer sealing of the aneurysm neck with one ICA stent mesh and two crushed stent meshes, may result in high metal coverage at the neck, leading to a flow diversion effect. High packing density has been reported to lead to a reduced aneurysm recurrence [10]. Therefore, the $\lambda$ stenting technique could safely achieve a low recurrence rate for fetal-type PCOM aneurysms without increasing perioperative complications.

\section{$\lambda$ stenting versus other stenting techniques and direct surgery}

Various complex stenting techniques for endovascular treatment of fetal-type PCOM aneurysms have been reported previously (Fig. 4) [11-13]. The present study indicates that the $\lambda$ stenting technique may be more effective than other stenting techniques. In PCOM stenting alone, it is difficult to definitively preserve the ICA because PCOM stenting does not cover the aneurysm neck. In the $T$ configuration stenting technique, it is difficult to definitively preserve the PCOM because the occurrence of gaps between the ICA and PCOM stenting cannot be completely eliminated. Accordingly, in these techniques, tight packing of aneurysms is difficult for definite preservation of the ICA and PCOM. Alternatively, in the $\lambda$ stenting technique, it is possible to definitively preserve the ICA and PCOM because both the ICA and PCOM stent meshes can protect the relevant arteries by utilizing the crush technique.

Flow diverters have been increasingly used in endovascular treatment of PCOM aneurysms as well as widenecked large and giant aneurysms. Several reports have demonstrated that flow diverters, such as the Pipeline Embolization Device (PED; Medtronic, Minneapolis, Minnesota), contribute to the effective rate of occlusion but carry a risk of PCOM occlusion [6, 14, 15]. PCOM occlusion following flow diverter deployment has not caused any neurological symptoms $[6,14,15]$. However, flow diverter treatment for fetal-type PCOM aneurysms has been associated with incomplete aneurysm occlusion 
Fig. 4 Schematic image of the various complex stenting techniques for fetal-type PCOM aneurysms. $\mathrm{T}$ configuration stenting (A), Y configuration stenting (kissing and crossing; $\mathbf{B}$ and $\mathbf{C}$, respectively) and PCOM stenting (D) are depicted. PCOM, posterior communicating artery
(A)

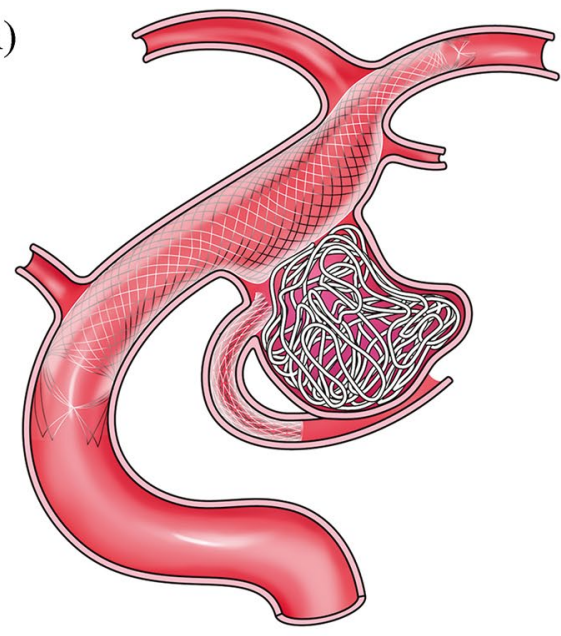

(C)

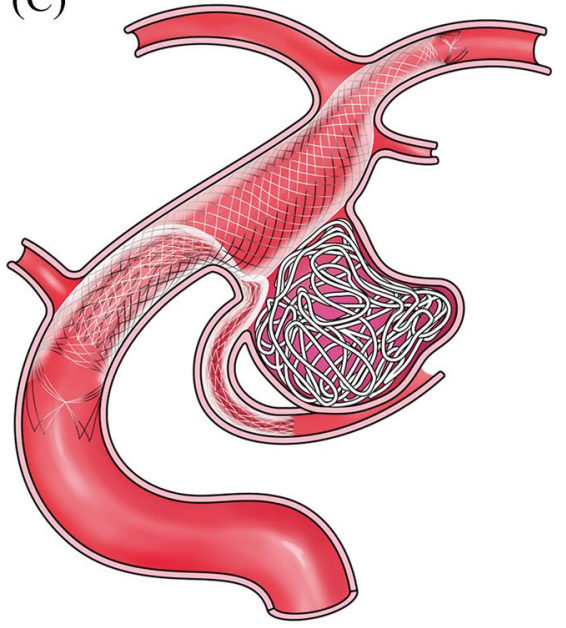

(B)

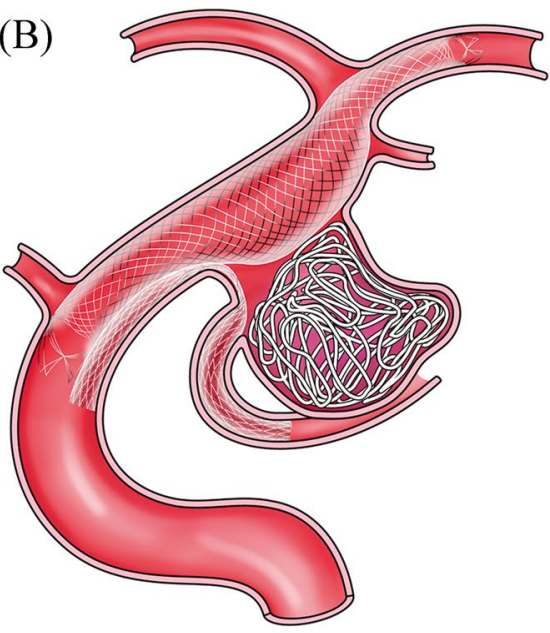

(D)

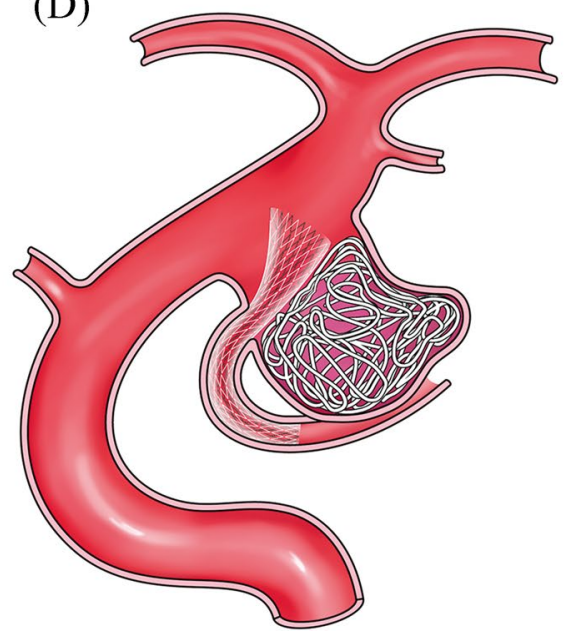

$[6,7]$. Therefore, endovascular coiling or surgical clipping is recommended for fetal-type PCOM aneurysms.

Surgical clipping has been considered beneficial for fetal-type PCOM aneurysms [4]. However, fetal-type PCOM aneurysms carry a greater risk of periprocedural ischemic injury even with surgical clipping [16]. Reconstruction clipping of the fetal-type PCOM aneurysm originating from the dome is even difficult when attempting to achieve complete obliteration. Although a superficial temporal artery and posterior cerebral artery bypass are performed, followed by complete aneurysm clipping that involves sacrificing the PCOM, a PCOM perforator infarction can be a valid concern. Furthermore, anatomical features of aneurysms, such as the adherent anterior choroidal artery, projection of aneurysms, and the surgical site, could cause surgical clipping-related complications [17-19]. In contrast, the $\lambda$ stenting technique could safely achieve a high packing density for the fetal-type PCOM aneurysms because the aforementioned anatomical features never influence the results of endovascular treatment.

\section{Technical tips for the endovascular procedure}

Catheterization of the PCOM arising from the dome of the aneurysm with a steep angle can be a difficult procedure. The order of PCOM catheterization, coiling, and stent deployment is variable in the clinical setting. The order of these procedures depends on the difficulty of PCOM catheterization due to a patient's unique vascular anatomy, such as tortuosity of the access route and orifice of the PCOM angle. In most cases, the PCOM catheter could be introduced with/ without balloon assistance having been previously placed in the ICA. In addition, in cases where primary PCOM cannulation is difficult, adjuvant techniques can be applied, such as half deployment of the ICA stent before PCOM cannulation. Accordingly, ICA stenting and balloon assistance could help PCOM cannulation by acting as a scaffold for microcatheters. For PCOM cannulation, we applied a preshaped microcatheter or a simple manual shape, such as a micro pigtail, for cannulation of the PCOM according to the anatomical variation. These shaped microcatheters enabled 
a steerable micro guidewire with a soft tip to directly select the PCOM without scratching the aneurysm surface.

To prevent PCOM stent-edge placement of the distal neck of the aneurysm from unintentionally migrating toward the aneurysm dome, we always prepare a coil frame as a scaffold with balloon assistance before PCOM stenting. Further, to deploy the PCOM stent in opening the distal flair forward distal neck of the aneurysms, a microcatheter for the PCOM stent needs to be slightly compressed when distal flair is deployed.

\section{Limitations}

A major drawback of the $\lambda$ stenting technique appears to be thromboembolic complications caused by the complex stenting procedure. Complex stenting techniques, such as Y-configuration stenting, have been reported to cause thromboembolic complications, but are considered to be acceptable $[11,12]$. The mesh coverage of the PCOM orifice in $\lambda$ stenting is almost the same as other complex stenting and flow diverter placement. Moreover, the metal surface exposed in the ICA flow is reduced compared to Y-configuration stenting. Hence, $\lambda$ stenting could be performed without major thromboembolic complications.

The small number of patients and the short follow-up period in this preliminary study may be inadequate to draw definite conclusions on long-term outcomes and adequate maintenance of antiplatelet agents. Therefore, further studies with longer follow-up periods are needed to evaluate these points.

\section{Conclusions}

The $\lambda$ stenting technique can enable a dense and stable coil packing of PCOM aneurysms with fetal-type PCOM originating from the aneurysm dome, while preserving the PCOM. The $\lambda$ stenting technique is considered safe and effective, as reported by this study. Therefore, it could become an alternative treatment option for this sub-type of intracranial aneurysms.

Supplementary Information The online version contains supplementary material available at https://doi.org/10.1007/s00234-021-02775-y.

Acknowledgements We express our gratitude to Drs Jumpei Oda, and Takeya Suzuki for their invaluable support of this study.

Author contribution JT and IN contributed conception and design of the study; JT organized the database; JT, SM, YS, JM, AH, SW, KS, $\mathrm{KK}$, and KI contributed to the acquisition and analysis of the data; JT wrote the first draft of the manuscript; JT and IN wrote sections of the manuscript. All authors contributed to manuscript revision, and read and approved the submitted version.
Funding This research did not receive any specific grant from funding agencies in the public, commercial, or not-for-profit sectors.

Data availability Not applicable

\section{Declarations}

Competing interests Ichiro Nakahara reports grants from Kaneka Medix, Medtronic, and Nipro, and consultancy from Kaneka Medix, and Medicos Hirata, outside the submitted work.

Ethics approval The present study is a retrospective review of medical charts and radiographic data. The study protocol was conducted in accordance with the principles of the Declaration of Helsinki and approved by the institutional ethics committee (HM 20-297).

Consent to participate The need for written informed consent for participation was waived in accordance with the national legislation and institutional requirements. On our institute's website, all participants were provided with the opportunity to opt out of this study.

Consent for publication Written informed consent for publication was obtained from the participants.

Open Access This article is licensed under a Creative Commons Attribution 4.0 International License, which permits use, sharing, adaptation, distribution and reproduction in any medium or format, as long as you give appropriate credit to the original author(s) and the source, provide a link to the Creative Commons licence, and indicate if changes were made. The images or other third party material in this article are included in the article's Creative Commons licence, unless indicated otherwise in a credit line to the material. If material is not included in the article's Creative Commons licence and your intended use is not permitted by statutory regulation or exceeds the permitted use, you will need to obtain permission directly from the copyright holder. To view a copy of this licence, visit http://creativecommons.org/licenses/by/4.0/.

\section{References}

1. Yasargil MG (1984) Internal carotid artery aneurysms. Microneurosurgery, vol II. Thieme Stratton Inc, New York, pp 33-123

2. Spetzler RF, McDougall CG, Zabramski JM et al (2015) The Barrow Ruptured Aneurysm Trial: 6-year results. J Neurosurg 123:609-617

3. Molyneux AJ, Kerr RSC, Yu LM, Clarke M, Sneade M, Yarnoid JASP (2005) International subarachnoid aneurysms trial (ISAT) of neurosurgical clipping versus endovascular coiling in 2143 patients with ruptured intracranial aneurysms. Lancet (London, England) 366:809-817

4. Golshani K, Ferrell A, Zomorodi A et al (2010) A review of the management of posterior communicating artery aneurysms in the modern era. Surg Neurol Int 1:88

5. Choi HH, Cho YD, Yoo DH et al (2020) Impact of fetal-type posterior cerebral artery on recanalization of posterior communicating artery aneurysms after coil embolization: matched-pair case-control study. J Neurointerv Surg 12:783-787

6. Roy AK, Howard BM, Haussen DC et al (2018) Reduced efficacy of the pipeline embolization device in the treatment of posterior communicating region aneurysms with fetal posterior cerebral artery configuration. Neurosurgery 82:695-700 
7. Rinaldo L, Brinjikji W, Cloft $\mathrm{H}$ et al (2019) Effect of fetal posterior circulation on efficacy of flow diversion for treatment of posterior communicating artery aneurysms: a multi-institutional study. World Neurosurg 127:e1232-e1236

8. Mascitelli JR, Moyle H, Oermann EK et al (2015) An update to the Raymond-Roy Occlusion Classification of intracranial aneurysms treated with coil embolization. J Neurointerv Surg $7: 496-502$

9. Ormiston JA, Currie E, Webster MWI et al (2004) Drug-eluting stents for coronary bifurcations: Insights into the crush technique. Catheter Cardiovasc Interv 63:332-336

10. Lanzino G, Kallmes DF (2013) REVIEW ARTICLE Review of 2 decades of aneurysm-recurrence literature, Part 1 : reducing recurrence after endovascular coiling. Ajnr Am J Neuroradiol 21-23. https://doi.org/10.3174/ajnr.A3032

11. Zhao K-J, Yang P-F, Huang Q-H et al (2012) Y-configuration stent placement (crossing and kissing) for endovascular treatment of wide-neck cerebral aneurysms located at 4 different bifurcation sites. AJNR Am J Neuroradiol 33:1310-6

12. Chen Z, Niu Y, Tang J et al (2015) Endovascular treatment of posterior communicating artery aneurysms in the presence of the fetal variant of posterior cerebral artery. Interv Neuroradiol 21:456-61

13. Cho YD, Lee WJ, Kim KM et al (2013) Stent-assisted coil embolization of posterior communicating artery aneurysms. AJNR Am J Neuroradiol 34:2171-2176

14. Daou B, Valle-Giler EP, Chalouhi N et al (2017) Patency of the posterior communicating artery following treatment with the Pipeline Embolization Device. J Neurosurg 126:564-569
15. Rangel-Castilla L, Munich SA, Jaleel N et al (2017) Patency of anterior circulation branch vessels after Pipeline embolization: longer-term results from 82 aneurysm cases. J Neurosurg 126:1064-1069

16. Zada G, Breault J, Liu CY et al (2008) Internal carotid artery aneurysms occurring at the origin of fetal variant posterior cerebral arteries: surgical and endovascular experience. Neurosurgery 63:55-62

17. Sanai N, Caldwell N, Englot DJ, Lawton MT (2012) Advanced technical skills are required for microsurgical clipping of posterior communicating artery aneurysms in the endovascular era. Neurosurgery 71:285-295

18. Tanabe J, Ishikawa T, Moroi J et al (2018) Impact of right-sided aneurysm, rupture status, and size of aneurysm on perforator infarction following microsurgical clipping of posterior communicating artery aneurysms with a distal transsylvian approach. World Neurosurg 111:e905-e911

19. Fukuda H, Hayashi K, Yoshino K et al (2016) Impact of aneurysm projection on intraoperative complications during surgical clipping of ruptured posterior communicating artery aneurysms. Neurosurgery 78:381-390

Publisher's note Springer Nature remains neutral with regard to jurisdictional claims in published maps and institutional affiliations. 